(2) Open Access Full Text Article

\title{
Assessment of the efficacy of first-line antimalarial drugs after 5 years of deployment by the National Malaria Control Programme in Côte d'lvoire
}

This article was published in the following Dove Press journal:

Open Access Journal of Clinical Trials

8 November 2011

Number of times this article has been viewed

\author{
Andre T Offianan' \\ Serge B Assi ${ }^{2}$ \\ Aristide MA Coulibaly' \\ Landry T N'guessan' \\ Aristide A Ako' \\ Florence K Kadjo 2 \\ Moïse K San ${ }^{2}$ \\ Louis K Penali²
}

'Malariology Department, Institut Pasteur de Côte d'Ivoire, Abidjan, Côte d'Ivoire; ${ }^{2}$ National Malaria

Control Programme, Abidjan, Côte d'Ivoire
Correspondence: Andre T Offianan Malariology Department, Institut Pasteur de Côte d'lvoire, PO Box 490,

Abidjan 0I, Côte d'lvoire

Tel +22522448425

Fax +225 22485305

Email andre_offianan@yahoo.fr
Background: The emergence of artemisinin resistance has raised concerns that the most potent antimalarial drug may be under threat. Artesunate + amodiaquine (ASAQ) and artemetherlumefantrine $(\mathrm{AL})$ are respectively the first- and second-line treatments for uncomplicated falciparum malaria in Côte d'Ivoire. A comparison of the efficacy and safety of these two drug combinations was necessary to make evidence-based drug treatment policies.

Methods: In an open-label, non inferiority, randomized, controlled clinical trial, children aged 6-59 months were randomized to receive ASAQ or AL. Both drug regimens were given for 3 days, and follow-up was for 28 days. The primary endpoint was the 28-day cure rates and was defined as proportion of patients with polymerase chain reaction (PCR)-corrected cure rate after 28 days of follow-up.

Findings: A total of 251 patients who were attending the Ayame and Dabakala hospitals and presenting with symptomatic acute uncomplicated falciparum malaria were randomized to receive ASAQ (128) and AL (123). The intention-to-treat analysis showed effectiveness rates of $94.5 \%$ and $93.5 \%$ for ASAQ and AL, respectively on day 28 . After adjustment for PCR results, these rates were $96.1 \%$ and $96.8 \%$, respectively. On day 28 , the per-protocol analysis showed effectiveness rates of $98.4 \%$ and $96.6 \%$ for ASAQ and AL, respectively. After adjustment by PCR for reinfection, these rates were $100 \%$ for each drug, and both regimens were well tolerated.

Conclusion: ASAQ and AL remain efficacious treatments of uncomplicated falciparum malaria in Ivorian children 5 years after adoption. The efficacy of ASAQ and AL in Côte d'Ivoire requires, therefore, continuous monitoring and evaluation.

Keywords: artesunate, amodiaquine, artemether-lumefantrine, ASAQ, AL

\section{Introduction}

Multidrug resistance of Plasmodium falciparum is a major health problem in many countries, and the number of drugs available that are effective and affordable is very low.

Currently, artemether-lumefantrine (AL) and artesunate-amodiaquine (ASAQ) are the only widely available drugs, which are recommended by most malaria endemic countries in the treatment of uncomplicated falciparum malaria. ${ }^{1}$ The emergence of artemisinin-resistant malaria along the Thai-Cambodian border, historically a site of emerging antimalarial-drug resistance, has provoked global alarm that the most valuable and effective antimalarial drug is in danger of being lost, triggering a campaign to identify and eradicate resistant parasite strains..$^{2-6}$ Other suspected foci have been identified in the Greater Mekong subregion but are not yet confirmed. ${ }^{7}$ The high persistence of substandard artemisinin combined therapies (ACTs) and inappropriate 
artemisinin monotherapies in the private sector, especially in sub-Saharan Africa (where many individuals buy their treatments privately), risks patient safety, and through drug resistance, places the future of malaria treatment at risk globally. ${ }^{8-11}$ Several of these ACTs are currently available in Côte d'Ivoire, where ASAQ and AL combinations are common.

AL offers excellent efficacy, but in high-transmission areas, the relatively short half-life of lumefantrine leads to a high incidence of new infections soon after therapy. ${ }^{12,13}$ ASAQ has shown a significantly lower efficacy than has AL in East Africa, ${ }^{14,15}$ presumably due to the high prevalence of amodiaquine-resistant parasites in this region.

As resistance to sulfadoxine-pyrimethamine (SP) increases and spreads across Africa, ${ }^{16-18}$ the effectiveness of the intermittent preventive treatment in pregnancy (IPTp) strategy may be compromised in many malaria-endemic countries. ${ }^{19}$ Clinical trials are being conducted to assess alternative drugs that could be used for IPTp in place of SP. Artemisinin-based IPT regimens represent a promising potential alternative to SP. The World Health Organization (WHO) endorses the use of ACT for patients in the second and third trimesters of pregnancy with acute uncomplicated falciparum malaria. ${ }^{1}$ Artemisinin resistance would be disastrous for global malaria control. In Côte d'Ivoire, ASAQ and AL have been recommended respectively in first- and second-line treatment since 2005 following earlier studies. ${ }^{20-22}$ But continued use of amodiaquine or artesunate monotherapies, persistence of substandard ACTs in the private sector, ${ }^{10-11,23}$ and resistance to artesunate and/or amodiaquine ${ }^{24}$ may jeopardize the future use of ASAQ and AL as an effective artemisinin-based combination therapy. The emergence of artemisinin resistance may also compromise the future use of artesmisinin derivatives as potential alternatives to SP for IPTp.

The main purpose of this study was to update the baseline data from two randomized clinical trial sites assessing the safety and efficacy of ASAQ fixed-dose combination tablets in comparison with a fixed-dose combination of AL, the two drug combinations recommended by the National Malaria Control Programme (Abidjan, Côte d'Ivoire) before deployment of these two ACTs in the 19 districts. The Global Fund (Round 6) has provided funding for ASAQ and AL in Côte d'Ivoire for the treatment of uncomplicated falciparum malaria in children less than 5 years old.

\section{Patients, materials, and methods Study design}

This study was a randomized, open-label, noninferiority clinical trial comparing ASAQ (Coarsucam ${ }^{\circledR}$; Sanofi-Aventis,
Paris, France) and AL (Artrin ${ }^{\circledR}$; Lic Pharma, Abidjan, Côte d'Ivoire). The study was carried out according to current WHO protocol. ${ }^{25}$

\section{Study area}

This trial was performed from November 2008 through February 2009 at two health care centers in Ayame (southern) and Dabakala (northern), Côte d'Ivoire. P. falciparum transmission is intense and perennial, with recrudescence during the rainy season. $P$. falciparum is the predominant malaria-causing species.

\section{Study population}

Children presenting at the site were screened for eligibility and invited to participate in the study if they met the following inclusion criteria: aged 6-59 months; body weight $\geq 5 \mathrm{~kg}$; a history of fever in the previous 24 hours or measured fever (axillary temperature $\geq 37.5^{\circ} \mathrm{C}$ ); monoinfection with $P$. falciparum, with density parasitemia in the range of 2000-200,000 asexual parasites per microliters of blood; no other cause of fever than suspected malaria; and no general danger signs or signs of severe and complicated falciparum malaria as per WHO guidelines, ${ }^{25}$ were able to take study drugs by the oral route, were able to attend clinic on stipulated days for follow-up, and if a parent or guardian provided written informed consent for the child to participate in the study.

Exclusion criteria consisted of: presence of severe and complicated malaria as defined by WHO; a mixed plasmodial infection, or concomitant disease masking assessment of the response to antimalarial treatment; intake of antimalarial drugs other than chloroquine within the past 7 days; and known hypersensitivity to any of the investigative combinations.

\section{Randomization and treatment}

Patients were randomly assigned to one of two treatment groups, namely ASAQ once daily intake and AL twice daily.

Treatment dosages were determined according to patient's body weight. Treatment duration was 3 days. Treatment with ASAQ (one tablet once daily for 3 days) was provided as $25 \mathrm{mg} / 67.5 \mathrm{mg}$ tablets for children who weighed 5-9 kg, $50 \mathrm{mg} / 135 \mathrm{mg}$ tablets for children who weighed $>9-18 \mathrm{~kg}$, and $100 \mathrm{mg} / 270 \mathrm{mg}$ tablets for children who weighed $>18 \mathrm{~kg}$.

Treatment in the AL group was provided as $20 \mathrm{mg} / 120 \mathrm{mg}$ tablets, given in a 3-day, 6-dose regimen. Children who weighed 5-15 kg received one tablet per dose, and children who weighed $>15-20 \mathrm{~kg}$ were given two tablets per dose. 
The first dose was taken at enrolment, the second dose 8 hours later on day 0 , and then two doses at 12-hourly intervals for the subsequent 2 days. All tablets were either swallowed whole or crushed with water. Yoghurt was then given to the children. The drug administration was supervised by the research team.

Randomization was done by an independent statistician using computer-allocated blocks of ten and was not stratified. Individual treatment allocations were contained inside consecutively numbered sealed envelopes, which were opened sequentially by a study investigator or clinical research coordinator after the decision to enroll a subject had been made by the study team. In the case where a participant vomited the first dose within 30 minutes of drug administration, a repeat full dose was re-administered. If vomiting persisted, the child was withdrawn from the study. A participant who was withdrawn due to vomiting received rescue medication according to the Malaria Control Programme guidelines.

\section{Blinding}

Although initial treatment allocation was blinded, administration of subsequent medication was not (ie, open-label). However, laboratory staff reading the malaria smears had no idea of the treatment received. All additional sample investigation such as polymerase chain reaction (PCR) genotyping was performed by individuals who were also blinded to the treatment allocation.

\section{Efficacy and safety assessment}

The patients returned on days $1,2,3,7,14,21$, and 28 to complete the drug administration and for clinical assessment, blood smears, and filter paper blood sample collection. Patients who failed to report at the clinic for the scheduled visit were followed to their residence by trial field workers.

They were also asked to return to the clinic on any other day if they had new complaints or any change in their condition. Clinical efficacy was assessed by grading the pre-existing clinical signs and combining them with the temperature values. The following clinical symptoms were assessed systematically: fever, perspiration, headache, chills, pain (specifying topography), jaundice, asthenia, dizziness, anorexia, skin fold, skin rash, hepatomegaly, pruritus. Splenomegaly was estimated according to the Hackett scale. ${ }^{26}$ Parasitological efficacy was based on asexual parasitemia and gametocyte carriage.

The classification of the therapeutic outcome was done according to the current 28-day WHO protocol. ${ }^{25}$ The primary endpoint was the 28-day cure rates and was defined as the proportion of patients with PCR-corrected adequate clinical and parasitological response (ACPR) after 28 days of follow-up. Secondary endpoints were clinical adverse events (AEs), clearance rate of fever and parasitemia, and gametocyte carriage.

All AEs were monitored passively and actively. An AE was defined as any unfavorable and unintended sign, symptom, or disease temporarily associated with the use of an investigational product, not present on day 0 , but occurring during follow-up, or was present on day 0 but became worse during follow-up.

\section{Sample size}

The sample size was calculated using the WHO guidelines on assessment of antimalarial drugs. ${ }^{25}$ The population size was determined according to the following criteria: the proportion of probable clinical failures with the antimalarial combinations studied should not be higher than $10 \%$, for a level of confidence of $95 \%$ and a precision of $10 \%$, taking into account patients who were excluded or lost to follow-up. Using these criteria, a minimum of 50 patients was required in each treatment arm per site.

\section{Laboratory procedures}

At enrolment and subsequent days of follow-up, thick and thin blood films were collected. Blood smears were stained with Giemsa $10 \%$ and were examined at magnification $100 \times$ (oil immersion). Density parasitemia was measured by counting the number of asexual parasites against a number of leukocytes in the thick blood film, based on a putative count of 8000 leukocytes per microliter of blood. The number of asexual parasites was counted against 200 leukocytes using a hand tally counter. If $P$. falciparum gametocytes were seen, a gametocyte count was performed against 1000 leukocytes. Counts were performed by two independent microscopists; discrepant readings were resolved by a third reader.

Blood spots on Whatman 3M filter paper were prepared for PCR genotyping. Multiplicity of infection was assessed using three polymorphic loci (merozoite surface proteins 1 and 2 and glutamate-rich). ${ }^{27}$ Briefly, filter paper blood samples collected on the day of enrolment and on the day of failure were analyzed for polymorphism of merozoite surface protein-1 ( $m s p-1)$ and merozoite surface protein-2 ( $m s p$-2) using nested-PCR as previously described. ${ }^{28}$ Possible outcomes were new infection or recrudescence. A "new infection" is a subsequent occurring parasitemia in which all the alleles in parasites from the post-treatment sample are different from those in the admission sample, for one or more loci 
tested. In a "recrudescence," at least one allele at each locus should be in common for both paired samples. ${ }^{27}$

\section{Statistical analysis}

Data generated were recorded in a log book and individual participants case record files. Data were entered and analyzed with EPI-Info ${ }^{\text {TM }}$ software (v 6.4; Centers for Disease Control and Prevention, Atlanta, GA). Analysis of treatment outcome was intention-to-treat (ITT) and per protocol.

Per-protocol analysis included all patients who matched all inclusion criteria, were properly randomized, had received the study drugs according to the protocol, and for whom data were available on the study endpoint without protocol violation during the follow-up time. ITT analysis comprised all randomized patients fulfilling the inclusion criteria without repeated vomiting after the first study drug administration.

Frequencies were compared by either chi-squared or Fisher's exact tests, as appropriate, and continuous variables by Student's $t$-tests when the data are normally distributed. No normally distributed data were transformed to normality.

\section{Ethical issues}

The study was conducted according to the Declaration of Helsinki and national legal and regulatory requirements. The protocol was submitted and approved by the national ethics committee. Written informed consent was obtained from the parent or guardian. If parents or guardian were unable to sign, a fingerprint was applied on the consent form. If the parents or guardian were unable to read, the informed consent form was read and explained in the appropriate language. In such cases, the presence of a witness who also signed the consent form to confirm that the patient had freely given consent was required.

\section{Results}

\section{Participant characteristics}

Between November 2008 and February 2009, out of 624 children under 5 years of age who were screened for

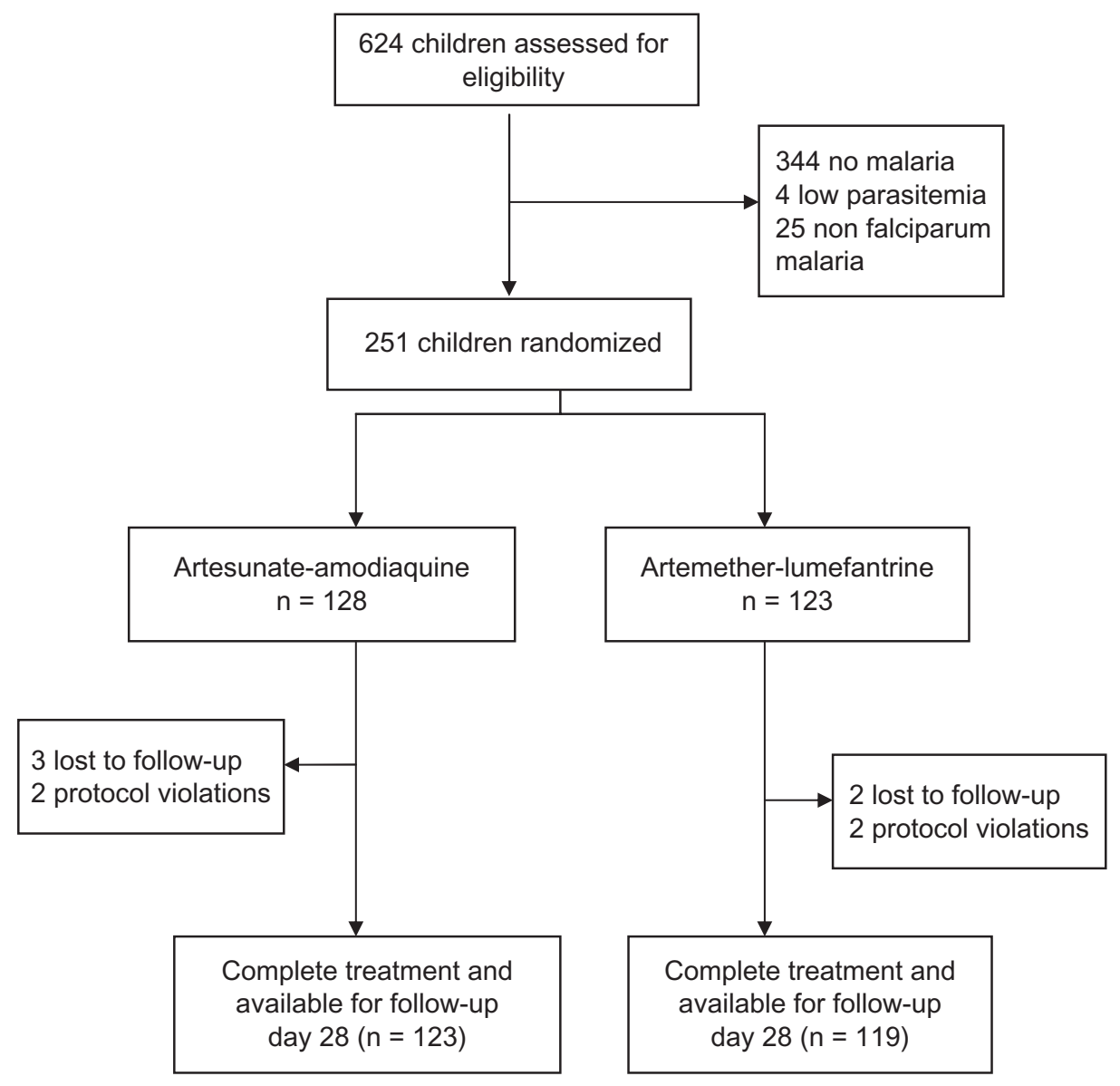

Figure I Trial profile. 
malaria symptoms, 251 were enrolled. Figure 1 shows detailed enrolment and follow-up information. Of the 251 enrolled participants, 128 were assigned to ASAQ and 123 to AL.

A total of 123 and 119 patients of the ASAQ and AL, respectively, completed the trial and had adequate data for the analysis of the endpoints. Table 1 shows the baseline characteristics of the eligible participants. The two treatment groups were comparable in all characteristics.

\section{Clinical and parasitological responses to treatment}

As shown in Table 2, the 28-day cure rates were 94.5\% (121 of 128 ) and $93.5 \%$ (115 of 123) for participants receiving ASAQ and AL, respectively $(P=0.73)$. After adjusting for cases of reinfection, the 28-day cure rates were 96.1\% (123 of 128 ) and $96.8 \%$ (119 of 123) for those receiving ASAQ and AL, respectively.

The per-protocol analysis showed effectiveness rates on day 28 of $98.4 \%$ and $96.6 \%$ for ASAQ and AL, respectively $(P=0.44)$. After adjustment by PCR for reinfection, these rates were $100 \%$ for each drug.

\section{Fever and parasite clearance and gametocyte carriage}

As shown in Figure 2, the proportion of participants without fever was similar on days 2 and 3. On day 2, the fever clearance rates were $77.2 \%$ (95 of 123) and $73.9 \%$ (88 of 119) in the ASAQ and AL groups, respectively $(P=0.55)$, while on day 3 , these rates were $82.1 \%$ (101 of 123$)$ and $81.5 \%$ (97 of 119).

Both treatments resulted in rapid clearance of parasites (Figure 3 ) as parasite clearance rates on day 2 were $100 \%$ (123 of 123) and 99.2\% (118 of 119) in the ASAQ and AL groups, respectively.

At enrolment, gametocytes were detected in the peripheral blood of eight patients: three in the ASAQ group and five in the AL group. On day 3, three patients of each group had gametocytes. All patients were free of gametocytes from day 7 to day 28 (Figure 4 ).

\section{PCR findings}

All six patients (two in the ASAQ group and four in the AL group) who were parasitemic during follow-up had their samples analyzed at enrolment and post-treatment. Parasite genotypes in the pre- and post-treatment samples were nonidentical in all patients and were considered as newly acquired infections.

\section{AEs}

Assessment of AEs was made difficult by the predominance of background signs and symptoms of malaria and the age of the participants. Common AEs recorded (ITT population) were vomiting, nausea, diarrhea, asthenia, and abdominal pains. All of these events were described as mild or moderate. Table 3 shows details of the distribution of AEs recorded during the study.

\section{Discussion}

Artemisinins are established antimalarial agents with an excellent safety profile, ${ }^{29}$ and ACTs are the recommended first-line treatments of falciparum malaria in all countries with endemic disease. ${ }^{1}$ There are recent concerns that the efficacy of such therapies has declined on the ThaiCambodian border. ${ }^{7,30,31}$ In Côte d'Ivoire, more than 100 combinations are registered and marketed and widely available on street markets as poor-quality byproducts. Parasite resistance to amodiaquine alone was also well established in most African countries. ${ }^{24,32,33}$ Overall, there are concerns about the efficacy of registered treatments, especially ASAQ.

In the present study, the efficacy and safety of ASAQ and $\mathrm{AL}$ in the treatment of acute uncomplicated malaria in Ivorian children aged 6-59 months were thus compared. ASAQ administered once daily for 3 days was as effective as the six-dose regimen of AL given twice daily for 3 days.

Table I Baseline characteristics in the intention-to-treat cohort

\begin{tabular}{lll}
\hline Characteristic & ASAQ & AL \\
\hline Mean age (range) & $31.24(6-59)$ & $34.4 I(7-59)$ \\
Female sex, $\mathrm{n}(\%)$ & $60(68)$ & $58(65)$ \\
Mean weight, $\mathrm{kg}$ (range) & $12.06(5.4-19.0)$ & $12.49(6.5-20.0)$ \\
Mean temperature, ${ }^{\circ} \mathrm{C}$ (range) & $38.6 \mathrm{I}(36-4 \mathrm{I})$ & $38.12(36.4-40.7)$ \\
GM parasite count, $\mu \mathrm{L}$ (range) & $30,487(2000-17 \mathrm{I}, 152)$ & $0.43^{\mathrm{b}}$ \\
\hline
\end{tabular}

Notes: achi-squared test; 'Student's t-test.

Abbreviations: ASAQ, artesunate + amodiaquine; AL, artemether-lumefantrine; GM, geometric mean. 
Table 2 Treatment outcome of ASAQ and AL at day 28

\begin{tabular}{|c|c|c|c|}
\hline Outcome & ASAQ & $\mathbf{A L}$ & $P$ value \\
\hline \multicolumn{4}{|l|}{ Intention to treat analysis } \\
\hline Enrolled patients & 128 & 123 & \\
\hline Patients seen at day 28 & $123 / 128(96.1 \%)$ & $119 / 123(96.8 \%)$ & I \\
\hline Missing & $5 / 128(3.9)$ & $4 / 123(3.2 \%)$ & I \\
\hline Crude failure rate at day 28 & $7 / / 28$ (5.5\%) & $8 / 123(6.5 \%)$ & 0.73 \\
\hline Crude cure rate at day 28 & $121 / 128(94.5)$ & $115 / 123(93.5)$ & 0.73 \\
\hline PCR adjusted failure rate at day 28 & $5 / 128(3.9 \%)$ & $4 / 123(3.2 \%)$ & I \\
\hline PCR adjusted cure rate at day 28 & $123 / 128(96.1 \%)$ & $119 / 123(96.8 \%)$ & 1 \\
\hline \multicolumn{4}{|l|}{ Per protocol analysis } \\
\hline Patients seen at day 28 & 123 & 119 & \\
\hline Crude failure rate at day 28 & $2 / 123(1.6 \%)$ & $4 / 119$ (3.4\%) & 0.44 \\
\hline Crude cure rate at day 28 & $121 / 123(98.4 \%)$ & $115 / 119(96.6 \%)$ & 0.44 \\
\hline PCR adjusted failure rate at day 28 & $0 / 123(0 \%)$ & $0 / 119(0 \%)$ & I \\
\hline PCR adjusted cure rate at day 28 & $123 / 123(100 \%)$ & $119 / 119(100 \%)$ & $\mathrm{I}$ \\
\hline
\end{tabular}

Note: $P$ values were obtained by Fisher's exact test.

Abbreviations: $\mathrm{AL}$, artemether-lumefantrine; $\mathrm{ASAQ}$, artesunate + amodiaquine; PCR, polymerase chain reaction.

The results showed a high cure rate for both regimens after a standard 28-day follow-up. These results are consistent with efficacy results reported from several sub-Saharan African countries, ${ }^{34-36}$ indicating a high overall cure rate with these regimens in the short term (28-day). Therefore, the data are in line with studies conducted in Ghanaian children showing lower parasitological and clinical failure rates in the ASAQ compared with the AL arm. ${ }^{37-39}$ In contrast, results from Central and East Africa show higher efficacy of AL than ASAQ, especially a higher potency in preventing reinfections. ${ }^{14,40}$ In the present study, both treatments were equivalent regarding parasite and fever clearance rates and gametocyte carriage. As already described, ${ }^{29}$ ACTs triggered a rapid asexual parasite clearance in the present cohort. Delay in parasite clearance ( $>2$ days) only occurred in one child of the AL group which could suggest decreased sensitivity. ${ }^{41}$ The results of this present study suggest full sensitivity of $P$. falciparum in vivo to ACTs in the area of study.

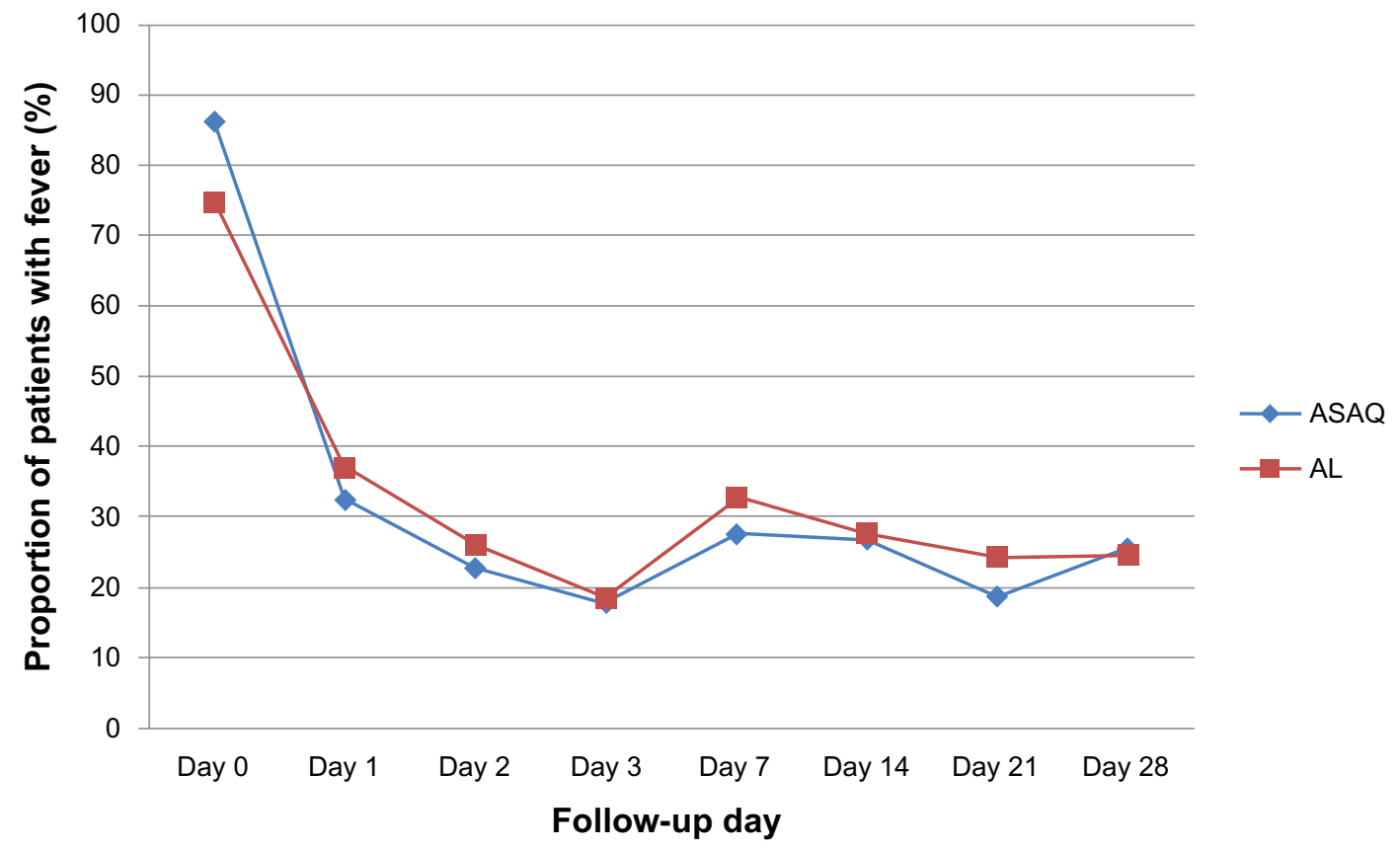

Figure 2 Fever clearance in the two groups.

Note: No significant differences were found for the proportions of subjects with fever between the two treatment regimens from baseline to day 28 ( $P>0.05$ Fisher's exact test or chi-squared test as appropriate).

Abbreviations: AL, artemether-lumefantrine; ASAQ, artesunate + amodiaquine. 


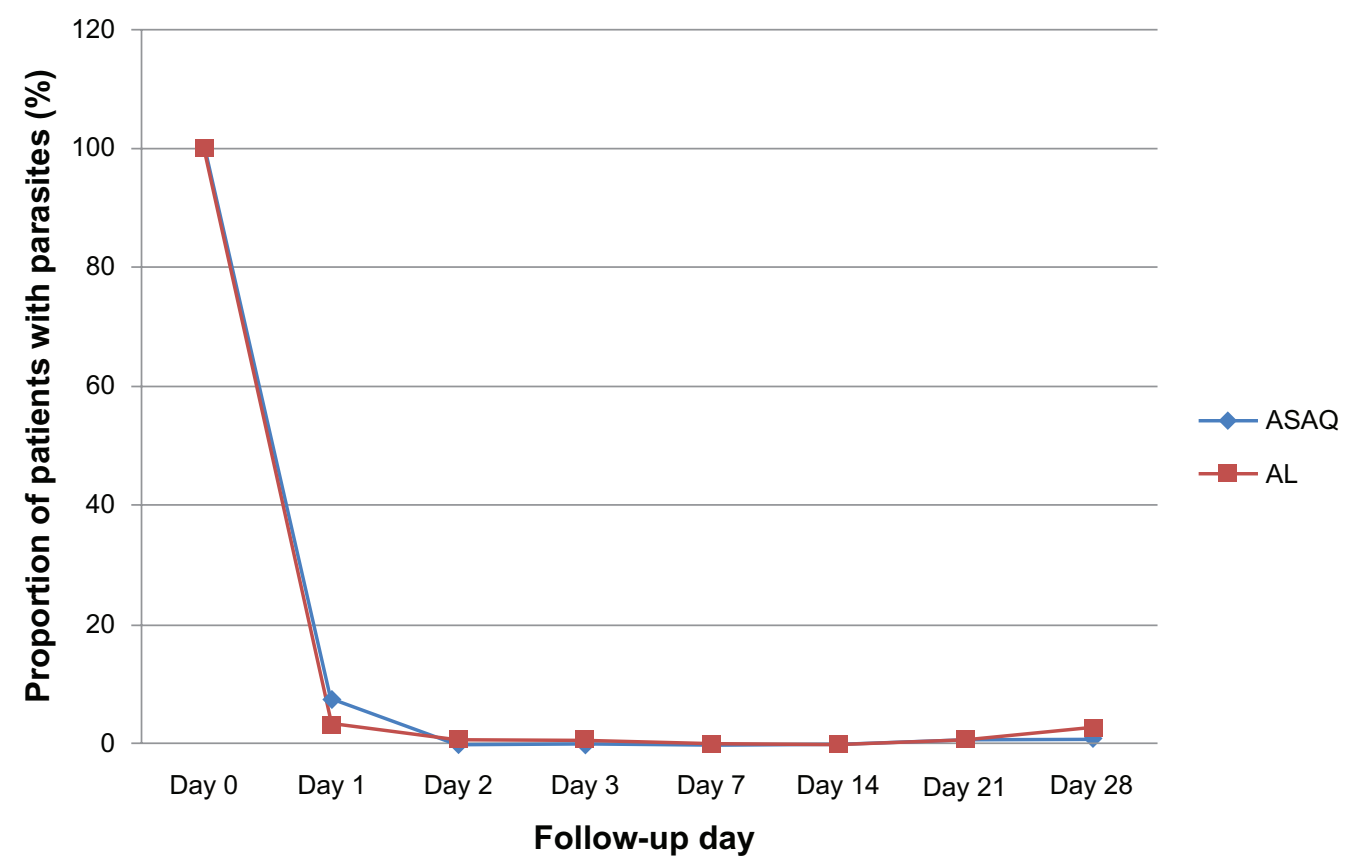

Figure 3 Parasite clearance in the two groups.

Note: The proportion of participants who were aparasitemic was similar between the two treatment arms: $100 \%$ and $99 \%(P=0.49)$ for those receiving ASAQ or AL, respectively, on day 2 . On day 3 these rates were also $100 \%$ ( 123 of 123$)$ and $99 \%$ (II8 of II9) in the ASAQ and AL groups respectively $(P=0.49)$.

Abbreviations: AL, artemether-lumefantrine; ASAQ, artesunate + amodiaquine.

A limitation of the study is the restriction of the follow-up period to 28 days bearing the risk of underestimation of treatment failures. Although logistically difficult, only longitudinal clinical trials with follow-up time over several months are appropriate for comparing the benefits and risks of different antimalarial drugs, especially in highly endemic areas. ${ }^{42}$ It has been estimated ${ }^{43}$ that 28 -day assessments underestimate the true failure by about $20 \%$ for drugs with intermediate elimination half-life, such as lumefantrine and SP (3-6 days half-life), ${ }^{44}$ and as much as $40 \%$ for drugs with

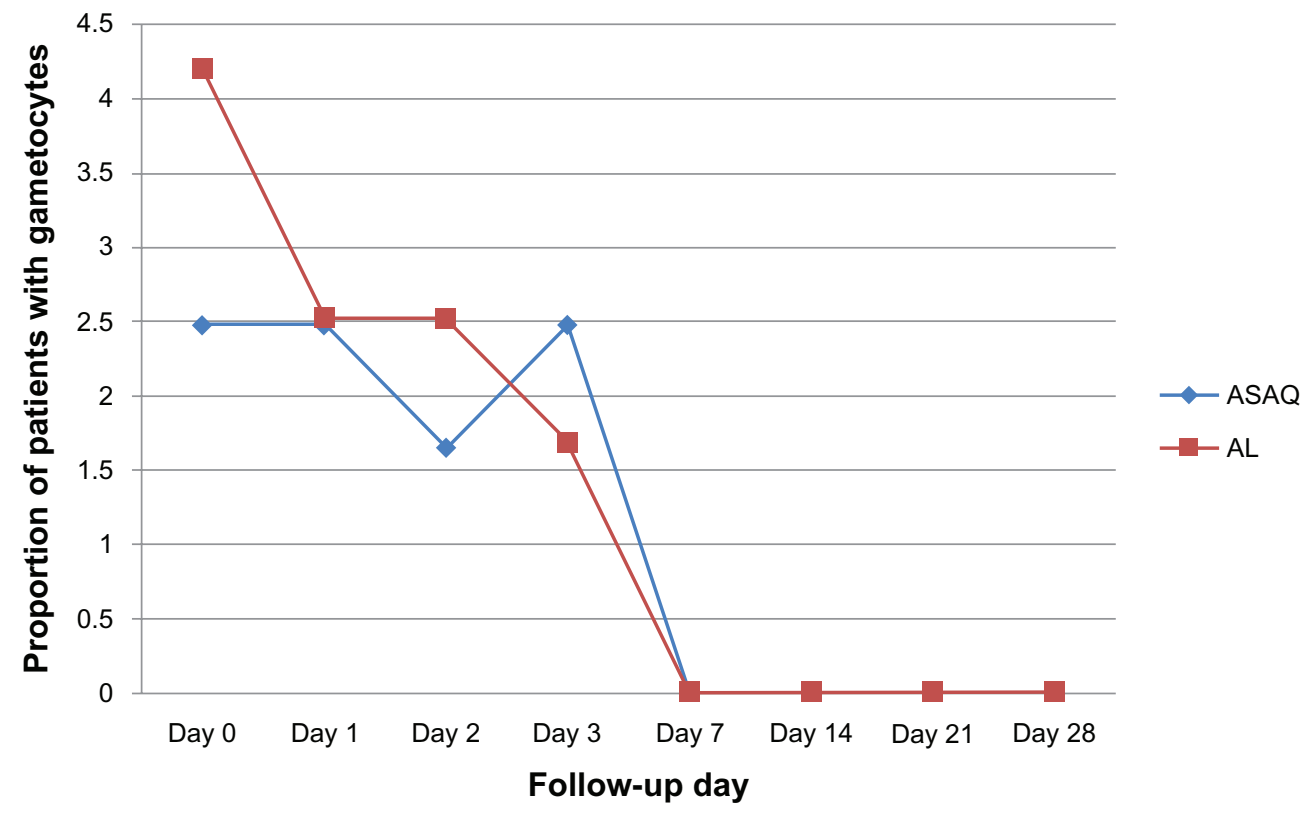

Figure 4 Proportion of patients with gametocytes during follow-up.

Abbreviations: AL, artemether-lumefantrine; ASAQ, artesunate + amodiaquine. 
Table 3 Clinical adverse events in the intention-to-treat population

\begin{tabular}{|c|c|c|c|c|c|}
\hline \multirow[t]{2}{*}{ Adverse event } & \multicolumn{2}{|l|}{ ASAQ } & \multicolumn{2}{|l|}{ AL } & \multirow[t]{2}{*}{$P$ value } \\
\hline & $\mathbf{N}=128$ & $\%$ & $N=123$ & $\%$ & \\
\hline Asthenia & 18 & 14.06 & 6 & 4.88 & 0.01 \\
\hline Anorexia & 5 & 3.91 & 2 & 3.25 & 0.45 \\
\hline Spitting & 12 & 9.38 & 7 & 5.69 & 0.27 \\
\hline Vomiting & 8 & 6.25 & 4 & 3.25 & 0.38 \\
\hline Diarrhea & 6 & 4.69 & 3 & 2.44 & 0.50 \\
\hline Abdominal pain & 10 & 7.81 & 4 & 3.25 & 0.17 \\
\hline Nausea & 15 & $1 \mathrm{I} .72$ & 10 & 8.13 & 0.34 \\
\hline
\end{tabular}

Notes: The most commonly reported and possibly drug-related adverse events to both combination therapies were gastrointestinal effects (abdominal pain, anorexia, diarrhea, and late vomiting); $P$ values were obtained by Fisher's exact test. Abbreviations: $\mathrm{AL}$, artemether-lumefantrine; ASAQ, artesunate + amodiaquine.

a longer elimination half-life, such as desethylamodiaquine, the active metabolite that amodiaquine quickly metabolizes into (1-3 weeks half-life). ${ }^{45}$ In this case, the true failure rates may have been higher. A trial comparing ASAQ and AL was performed in Ghana with a follow-up time of 1 year showing high day-28 ACPRs of $95.3 \%$ and $94.2 \%$, respectively, with no difference in the occurrence of reinfections or AEs. ${ }^{46}$ However, the differentiation between reinfection and resistance is even more difficult with increasing follow-up periods.

In the present study, the most common AEs were vomiting, nausea, diarrhea, asthenia, and abdominal pains. These are common clinical symptoms among patients with malaria. It is thus difficult to confidently classify them as drug-related AEs.

Both ACTs were not only efficacious but well tolerated. No study participant was withdrawn as a result of recurrent vomiting. In addition, there was no incidence of any serious $\mathrm{AE}$ in the two treatment groups. The incidence of gastrointestinal AEs was higher in the ASAQ group compared with the AL group. These findings are in agreement with previous drug safety monitoring. ${ }^{32-34}$ This may suggest a lower intrinsic tolerance of ASAQ.

Another limitation of this study is its limited statistical power to detect rare side effects, as well as the difficulties associated with evaluating neurological function in children below 5 years of age.

The two ACTs showed a good safety profile and were well tolerated. ASAQ and AL remain efficacious treatments of uncomplicated falciparum malaria in Ivorian children, and significant drug resistance does not seem to have emerged after 5 years of adoption as first- and second-line treatments. The Global Fund has provided funding for ASAQ and AL in Côte d'Ivoire to treat uncomplicated falciparum malaria in children. Correct deployment of ACTs remains now a major challenge.

\section{Authors' contributions}

All authors participated in the design, implementation, and analysis or data interpretation of the study. OAT and AS were involved in all phases of the study and have full access to all the data in the study. LKP and SK were the principal investigators and supervised the study. CMA an NTL were responsible for conduction of field studies and coordination of study procedures. Analysis of data was led by AAA. The manuscript was drafted by OAT; substantial input came from all investigators. All authors critically reviewed the report and approved the final version of the report for submission.

\section{Acknowledgments}

The authors thank the patients and their parents and guardians who agreed to participate in this study, and also thank all health authorities in the study sites for collaboration.

The assistance of the following personnel is gratefully acknowledged for their assistance during the trial: Drs Pode, Offo, and Dafless from Dabakala and Drs Diokouri Anne, Kouame, and M'Bo Jeanne from Ayame. The authors would also like to acknowledge the contributions of Drs Ronan Jambou and Demba Sarr who provided writing assistance, Mr Ghiorghis Belai for revision of the manuscript, and Mrs Gbaguidi Martin and Kobenan Kra who undertook central reading of the slides.

\section{Disclosure}

The Global Fund sponsored this trial. The authors report no conflicts of interest in this work.

\section{References}

1. World Health Organization (WHO). Guidelines for the Treatment of Malaria. 2nd ed. Geneva, Switzerland: WHO; 2010. Available from: http://whqlibdoc.who.int/publications/2010/9789241547925_eng.pdf. Accessed September 10, 2010

2. Enserink M. Malaria's drug miracle in danger. Science. 2010;328(5980): 844-846.

3. White NJ. Artemisinin resistance - the clock is ticking. Lancet. 2010; 376:2051.

4. World Health Organization(WHO). Global Report on Antimalarial Efficacy and Drug Resistance: 2000-2010. Geneva, Switzerland: WHO; 2010. Available from: http://who.int/malaria/publications/ atoz/9789241500470/ en/index.html. Accessed May 14, 2011.

5. Dondorp AM, Yeung S, White L, et al. Artemisinin resistance: current status and scenarios for containment. Nat Rev Microbiol. 2010;8(4): 272-280.

6. Dondorp AM, Nosten F, Yi P, et al. Artemisinin resistance in Plasmodium falciparum malaria. $N$ Engl J Med. 2009;361(5):455-467. 
7. World Health Organization (WHO). Global Plan for Artemisinin Resistance Containment (GPARC). Geneva, Switzerland: WHO; 2011. Available from: http://www.who.int/malaria/publications/atoz/artemisinin_resistance_containment_2011.pdf. Accessed May 29, 2011.

8. Newton PN, Fernández FM, Green MD, Primo-Carpenter J, White NJ. Counterfeit and substandard anti-infectives in developing countries. Antimicrobial Resistance in Developing Countries. 2010;(Pt 4): 413-443.

9. Goodman C, Brieger B, Unwin A, et al. Medicine sellers and malaria treatment in sub-Saharan Africa: what do they do and how can their practice be improved? Am J Trop Med Hy. 2007;77(6):203-218.

10. Granado S, Obrist B, Manderson L, Tanner M. The moment of sale: treating malaria in Abidjan, Côte d'Ivoire. Anthropol Med. 2009;16(3): 319-331

11. Aka E, Legris C, Tanimoto T, Matsushita R, Kimura K. Counterfeit medicine detection by authenticity investigation: a pilot study in the street market of Côte d'Ivoire. Jpn Soc Soc Pharm. 2005;24:7-16.

12. Kamya MR, Yeka A, Bukirwa H, et al. Artemether-lumefantrine versus dihydroartemisinin-piperaquine for treatment of malaria: a randomized trial. PLoS Clin Trials. 2007;2:e20.

13. Yeka A, Dorsey G, Kamya MR, et al. Artemether-lumefantrine versus dihydroartemisinin-piperaquine for treating uncomplicated malaria: a randomized trial to guide policy in Uganda. PLoS One 2008;3:e2390.

14. Dorsey G, Staedke S, Clark TD, et al. Combination therapy for uncomplicated falciparum malaria in Ugandan children: a randomized trial. JAMA. 2007;297:2210-2219.

15. Mutabingwa TK, Anthony D, Heller A, et al. Amodiaquine alone, amodiaquine + sulfadoxine-pyrimethamine, amodiaquine + artesunate and artemether-lumefantrine for outpatient treatment of malaria in Tanzanian children: a four-arm randomised effectiveness trial. Lancet. 2005;365:1474-1480.

16. Harrington WE, Mutabingwa TK, Muehlenbachs A, et al. Competitive facilitation of drug-resistant Plasmodium falciparum malaria parasites in pregnant women who receive preventive treatment. Proc Natl Acad Sci U S A. 2009;106:9027-9032.

17. Mockenhaupt FP, Bedu-Addo G, Eggelte TA, et al. Rapid increase in the prevalence of sulfadoxine-pyrimethamine resistance among Plasmodium falciparum isolated from pregnant women in Ghana. $J$ Infect Dis. 2008;198:1545-1549.

18. Vallely A, Vallely L, Changalucha J, Greenwood B, Chandramohan D. Intermittent preventive treatment for malaria in pregnancy in Africa: what's new, what's needed? Malaria J. 2007;6:16.

19. Tagbor H, Bruce J, Agbo M, Greenwood B, Chandramohan D. Intermittent screening and treatment versus intermittent preventive treatment of malaria in pregnancy: a randomised controlled non-inferiority trial. PLoS One. 2010;5(12):e14425.

20. Faye B, Offianan AT, Ndiaye JL, et al. Efficacy and tolerability of artesunate-amodiaquine (Camoquin plus) versus artemether-lumefantrine (Coartem) against uncomplicated Plasmodium falciparum malaria: multisite trial in Senegal and Ivory Coast. Trop Med Int Health. 2010;15(5):608-613

21. Offianan AT, Penali LK, Yapi JD, et al. A comparative, randomized clinical trial of artemisinin/naphtoquine twice daily one day versus artemether/lumefantrine six doses regimen in children and adults with uncomplicated falciparum malaria in Côte d'Ivoire. Malaria $J$. 2009;8:148.

22. Ministry of Health/National Malaria Control Programme: Malaria policies and strategies in Côte d'Ivoire. Revised document, February 2008.

23. Tipke M, Diallo S, Coulibaly B, et al. Substandard anti-malarial drugs in Burkina Faso. Malaria J. 2008;7:95.

24. Yavo W, Bla KB, Djaman AJ, et al. In vitro susceptibility of Plasmodium falciparum to monodesethylamodiaquine, quinine, mefloquine and halofantrine in Abidjan (Côte d'Ivoire). Afr Health Sci. 2010;10(2): $111-116$.
25. World Health Organization (WHO). Assessment and Monitoring of Antimalarial Drug Efficacy for the Treatment of Uncomplicated Falciparum Malaria. Volume 50. Geneva, Switzerland: WHO; 2003:1-68.

26. World Health Organization (WHO). Malaria Terminology. Report of a drafting committee appointed by the World Health Organization. Geneva, Switzerland: WHO Monograph Series; 1953:82.

27. World Health Organization (WHO). Methods and Techniques for Clinicals Trials on Antimalarial Drug Efficacy: genotyping to identify parasites population. Informal consultation organized by the Medicines for Malaria Venture and cosponsored by WHO; May 29-31, 2007; Amsterdam, The Netherlands.

28. Cattamanchi A, Kyabayinze D, Hubbard A, Rosenthal PJ, Dorsey G. Distinguishing recrudescence from reinfection in a longitudinal antimalarial drug-efficacy study: comparison of results based on genotyping of msp-1, msp-2, and glurp. Am J Trop Med Hy. 2003;68: $133-139$.

29. White NJ. Qinghaosu (artemisinin): the price of success. Science. 2008:320:330-334.

30. Bethell D, Se Y, Lon C, et al. Artesunate dose escalation for the treatment of uncomplicated malaria in a region of reported artemisinin resistance: a randomized clinical trial. PLoS One. 2011;6(5):e19283.

31. Noedl H, Se Y, Sriwichai S, et al. Artemisinin resistance in Cambodia: a clinical trial designed to address an emerging problem in Southeast Asia. Clin Infect Dis. 2010;51:e82-e89.

32. Tekete M, Djimde AA, Beavogui AH, et al. Efficacy of chloroquine, amodiaquine and sulphadoxine-pyrimethamine for the treatment of uncomplicated falciparum malaria: revisiting molecular markers in an area of emerging AQ and SP resistance in Mali. Malaria J. $2009 ; 8: 34$

33. Tinto H, Guekoun L, Zongo I, Guiguemde RT, D'Alessandro U, Ouedraogo JB. Chloroquine-resistance molecular markers (Pfcrt T76 and Pfmdr-1 Y86) and amodiaquine resistance in Burkina Faso. Trop Med Int Health. 2008;13:238-240.

34. Gbotosho GO, Sowunmi A, Happi CT, Okuboyejo TM. Therapeutic efficacies of artemisinin-based combination therapies in Nigerian children with uncomplicated falciparum malaria during five years of adoption as first-line treatments. Am J Trop Med Hyg. 2011;84(6): 936-943.

35. Ndiaye JL, Randrianarivelojosia M, Sagara I, et al. Randomized, multicentre assessment of the efficacy and safety of ASAQ - a fixed-dose artesunate-amodiaquine combination therapy in the treatment of uncomplicated Plasmodium falciparum malaria. Malaria J. 2009;8:125.

36. Adjei GO, Kurtzhals JA, Rodrigues OP, et al. Amodiaquine-artesunate vs artemether-lumefantrine for uncomplicated malaria in Ghanaian children: a randomized efficacy and safety trial with one year follow-up. Malaria J. 2008;7:127.

37. Owusu-Agyei S, Asante KP, Owusu R, et al. An open label, randomised trial of artesunate + amodiaquine, artesunate + chlorproguanil-dapsone and artemether-lumefantrine for the treatment of uncomplicated malaria. PLoS One. 2008;3:e2530.

38. Kobbe R, Klein P, Adjei S, et al. A randomized trial on effectiveness of artemether-lumefantrine versus artesunate plus amodiaquine for unsupervised treatment of uncomplicated Plasmodium falciparum malaria in Ghanaian children. Malaria J. 2008;7:261.

39. Koram KA, Abuaku B, Duah N, Quashie N. Comparative efficacy of antimalarial drugs including ACTs in the treatment of uncomplicated malaria among children under 5 years in Ghana. Acta Trop. 2005;95:194-203.

40. Kabanywanyi AM, Mwita A, Sumari D, Mandike R, Mugittu K, Abdulla S. Efficacy and safety of artemisinin-based antimalarial in the treatment of uncomplicated malaria in children in southern Tanzania. Malaria J. 2007;6:146.

41. Carrara VI, Zwang J, Ashley AA, et al. Changes in the treatment responses to artesunate-mefloquine on the northwestern border of Thailand during 13 years of continuous deployment. PLoS One. 2009;4:e4551. 
42. Laufer MK, Djimdé AA, Plowe CV. Monitoring and deterring drug-resistant malaria in the era of combination therapy. Am J Trop Med Hyg. 2007;77:160-169.

43. Stepniewska K, Taylor WR, Mayxay M, et al. In vivo assessment of drug efficacy against Plasmodium falciparum malaria: duration of follow-up. Antimicrob Agents Chemother. 2004;48:4271-4280.

44. Bloland PB. Drug Resistance in Malaria. Geneva, Switzerland: World Health Organization; 2001.
45. Krishna S, White NJ. Pharmacokinetics of quinine, chloroquine and amodiaquine. Clinical implications. Clin Pharmacokinet. 1996;30:263-299.

46. Badoe EV, Lamptey R, Goka BQ. Amodiaquine-artesunate vs artemether-lumefantrine for uncomplicated malaria in Ghanaian children: a randomized efficacy and safety trial with one year follow-up. Malaria J. 2008;7:127.

\section{Publish your work in this journal}

The Open Access Journal of Clinical Trials is an international, peerreviewed, open access journal publishing original research, reports, editorials, reviews and commentaries on all aspects of clinical trial design, management, legal, ethical and regulatory issues, case record form design, data collection, quality assurance and data auditing methodologies. The manuscript management system is completely online and includes a very quick and fair peer-review system, which is all easy to use. Visit http://www.dovepress.com/testimonials.php to read real quotes from published authors.

Submit your manuscript here: http://www.dovepress.com/open-access-journal-of-clinical-trials-journal 